\title{
Central-place foraging by rats on the radial maze: The effects of patch size, food distribution, and travel time
}

\author{
MARLA ASH and WILLIAM A. ROBERTS \\ University of Western Ontario, London, Ontario, Canada
}

\begin{abstract}
Rats foraged on a four-arm radial maze with one, two, three, and four food items (0.65-g pieces of cheese) placed on different arms (patches) of the maze. In two experiments, the hypothesis was tested that rats should carry food to the center of the maze more often when a patch contains one food item than when it contains multiple food items. Support for this prediction was found when the tendency to carry initial items encountered in patches was compared among the differentsized patches. However, a further observation failed to support the hypothesis: Food carrying declined from first to last item encountered in multiple-item patches with clustered food items. Experiment 1 revealed that food carrying was reduced when travel time was increased by barriers placed at arm entrances. Both Experiments 1 and 2 indicated that the tendency for rats to carry food to the center of the radial maze increased as the distance of food encountered on an arm increased from the center. In both experiments, some rats dealt with the problem of multiple items by resorting to multiple-item loading, and some rats carried food items from the end of an arm to a point on the arm nearer the center for consumption.
\end{abstract}

Many animals encountering a patch of food carry food items to a place of safety for consumption. This behavior has often been noted in wild rodents, including gray squirrels (Lima, Valone, \& Caraco, 1985), roof rats (Flannelly, Kemble, \& Hori, 1986), and Norway rats (Neider, Cagnin, \& Parisi, 1982). Lima et al. (1985) found that food carrying in gray squirrels was strongly controlled by food size, with large items frequently carried to cover and small items eaten in the patch.

As a theoretical account of this finding, Lima et al. (1985) suggested that gray squirrels adopt an optimal foraging strategy that involves a tradeoff between foraging efficiency and risk of predation. Although carrying food to safety minimizes an animal's exposure to predation and food thievery from other animals, repeatedly carrying food items to safety consumes time and energy and thus reduces foraging efficiency. Thus, small food items that can be eaten rapidly are consumed in the open with minimal risk of predation, but large items that take longer to consume are carried home to be eaten in safety.

Recent experiments carried out in our laboratory suggest that a number of central-place foraging strategies seen in wild rodents can be found in laboratory rats foraging on a radial maze. When Phelps and Roberts (1989) placed food items of varying size and weight on the ends of the arms of a four-arm radial maze, they found that large food items were almost always carried to the center of the maze

This research was supported by an operating grant from the National Sciences and Engineering Research Council of Canada to W. A. Roberts. Requests for reprints should be sent to W. A. Roberts, Department of Psychology, University of Western Ontario, London, ON, Canada N6A 5C2. for consumption but small food items were eaten on the arms. Time measurements of foraging components revealed that rats traveled faster on the arms of the maze when carrying food than when not carrying food and that rats ate food items more rapidly on the maze arms than in the center. These observations, which parallel those made of gray squirrels in a more natural setting (Lima et al., 1985), suggest that the center of the radial maze functions as a place of safety. Further research indicates that central-place foraging can be disrupted either by modifying the structure of the maze or by selected brain lesions. Experiments suggest that the intersection of multiple alleys draws food-carrying rats to the center of the maze because it affords a number of escape routes. When escape routes were placed on the ends of maze arms, food carrying was reduced substantially (Roberts, Phelps, \& Schacter, in press). Finally, recent research indicates that the mediodorsal nucleus of the thalamus may be an important brain structure involved in the control of centralplace foraging. Lesions in this region, but not in other brain structures, lowered the tendency to carry food items to the center of the radial maze (Schacter, Phelps, Brodbeck, Mogenson, \& Roberts, 1991).

The experiments reported here deal with an important question raised by previous radial maze studies of centralplace foraging. In all previous studies, only one piece of food has been placed on the end of each maze arm. The Lima et al. model (1985) makes the strong quantitative prediction that an optimal forager should always carry a single or last item in a patch to its home base. Because no return trip to obtain further food is necessary, the last item in a patch should always be carried, because it min- 
imizes predation risk and does not reduce foraging efficiency by adding extra travel time. However, gray squirrels do not always carry the last item in a patch to safety (Lima et al., 1985), and rats often eat smaller single items on the arms of a radial maze (Phelps \& Roberts, 1989). This observation suggests that animals may not conceptualize a food item as being the last one in a patch and/or compute the optimality of carrying that item.

In the present experiments, a weaker qualitative prediction from the Lima et al. (1985) model was tested. The model predicts that food carrying should drop from one food item to two or more food items in a patch. If a forager can carry only one food item at a time, a decision to carry one item while one or more other items remain in the patch amounts to a further decision to make a return visit to the patch. If animals tend to increase efficiency by reducing return trips to a patch, the tendency to eat items in the patch should increase as the number of items in the patch increases from one to two or more.

\section{EXPERIMENT 1}

Each arm of a four-arm radial maze contained a different number of food items-one, two, three, or four cubes of cheese weighing $0.65 \mathrm{~g}$. This weight was chosen because items of this weight were carried about $50 \%$ of the time in the Phelps and Roberts (1989) experiments. A comparison based on food-carrying behavior in arms or patches indicates the influence of number of items in the patch on decisions regarding food carrying.

Two other variables were studied in this experiment, because it was felt that they might affect the steepness of the gradient relating probability of food carrying to patch size. One variable was the distribution of food within patches. In two groups, food items were distributed along the arms, and in two other groups, food was clustered at the ends of the arms, as is depicted in the left-hand and center mazes shown in Figure 1. If increasing the number of items in a patch decreases the tendency to carry food items, the function depicting this decrease may be steeper with clustered food than with distributed food, because the presence of multiple items should be more per- ceptible when items are close to one another than when they are spread apart.

The final variable manipulated was the travel time involved in carrying food to the center of the maze. Barriers were placed at the entrances to maze arms for one group in each food distribution condition, and no barriers were used with the other two groups in each condition. Introducing barriers leads to a significantly longer travel time on the arms of the maze relative to the absence of barriers and significantly reduces food carrying (Phelps \& Roberts, 1989, Experiment 3). Because the introduction of barriers increases the energy and time expended on each visit to a patch, it was anticipated that the curve relating food-carrying probability to patch size might be steeper with barriers on the maze than with barriers absent. In other words, a forager encountering multiple food items in a patch should decide to eat more of those items in the patch when the cost of carrying each item to safety is high than when the cost of carrying is low.

\section{Method}

Subjects. The subjects were 24 male Long-Evans hooded rats (Rattus norvegicus) that were 100-120 days old at the start of the experiment. The rats were housed individually and were exposed to a 16:8-h light:dark schedule, with light onset at $600 \mathrm{~h}$ and offset at $2200 \mathrm{~h}$. Testing was performed between 1200 and $1700 \mathrm{~h}$, 6 days a week. All subjects were kept at approximately $85 \%$ of freefeeding weight.

Apparatus. The apparatus was a four-arm radial maze constructed of plywood and painted black. The four arms radiated from a circular central platform, with a $90^{\circ}$ angle between adjacent arms. The central platform was $35 \mathrm{~cm}$ in diameter, and each arm was $76 \mathrm{~cm}$ long $\times 9 \mathrm{~cm}$ wide. Both the center and the arms were open and contained no walls. Pieces of wooden dowling supported the arms and central platform at a height of $60 \mathrm{~cm}$ above the floor. Wooden barriers, $12.5 \mathrm{~cm}$ wide $\times 15 \mathrm{~cm}$ high $\times 5 \mathrm{~cm}$ deep, could be placed at the entrances to each of the four arms. Bolts attached to the barriers were inserted through small holes in the maze arms, allowing the barrier to be fixed to the arm with a wing nut.

The maze was located in a $3.1 \mathrm{~m} \times 3.1 \mathrm{~m}$ room, lighted by two enclosed overhead fluorescent lighting units. A blind was placed adjacent to the maze. It was made of gray posterboard mounted on a wooden frame, and it measured $111 \mathrm{~cm}$ wide $\times 145 \mathrm{~cm}$ high. The experimenter sat behind the blind and observed the animals' behavior through several small openings in the blind. A desk con-

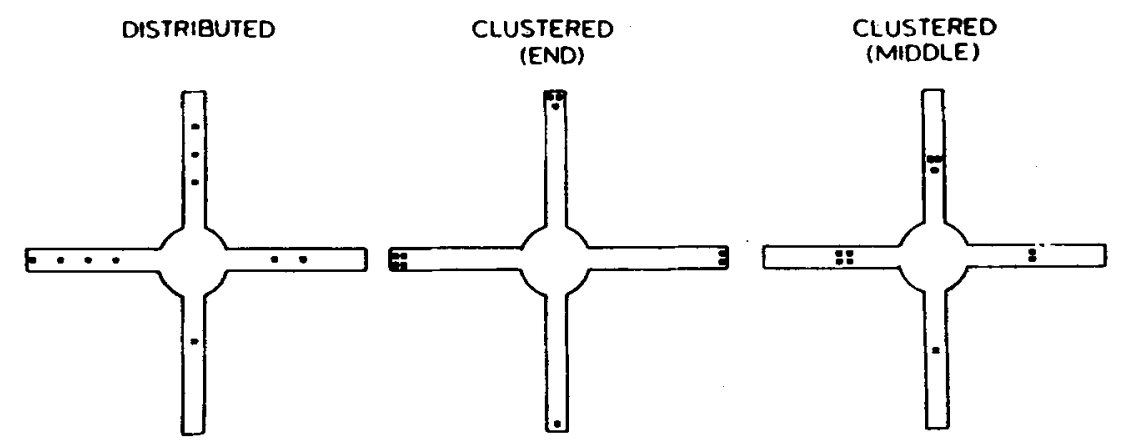

Figure 1. Top view of the four-arm radial maze. The filled squares represent the locations of food items in the distributed condition and in the end and middle clustered conditions. 
taining a Commodore 64 computer, a monitor, a printer, and a disk drive was situated beside the experimenter. White noise was played from an overhead speaker throughout the experimental sessions.

Procedure. Preliminary training lasted 5-7 days. Small pieces of Kraft American process (mild cheddar) cheese (.05-.20 g) were placed on the arms of the maze, and the subjects learned to run down the arms for cheese within a few days. Twelve randomly chosen rats were trained to climb over wooden barriers. Initially, the barriers were placed face down, then on their sides, and finally in the full upright position. Testing began when these animals were reliably scaling the barriers at the full height.

Each rat was tested for 20 sessions, with 1 session per day. In each session, one, two, three, and four 0.65 -g cubes of cheese were placed on different arms of the maze. The assignment of number of cubes of cheese to particular arms stayed constant throughout testing for each animal, but the assignments of number of items to arms varied between subjects. Six of the rats tested with barriers and 6 of the rats tested with no barriers were randomly assigned to the distributed food condition shown in the left-hand diagram in Figure 1. The initial food item was encountered $40 \mathrm{~cm}$ from the center, and subsequent items were spaced $12 \mathrm{~cm}$ apart. These two groups were designated barriers-distributed and no barriersdistributed. The other two groups of 6 rats were tested with food items clustered together at the end of each arm, as shown in the central diagram of Figure 1. These two groups were designated barriers-clustered and no barriers-clustered.

At the start of each session, the subject was placed in the center of the maze, and the experimenter then stepped behind the blind. The experimenter entered appropriate codes into the computer for various foraging behaviors. These codes indicated the location of each item and whether the item was eaten on the arm, carried to the center to be eaten, or partially carried. Partial carrying occurred when subjects carried the food item part of the way down an arm but consumed it before reaching the center.

All statistical tests reported were considered significant if $p<.05$.

\section{Results}

Food carrying. The tendency to carry food items varied between groups and between food items within a patch.
In virtually all cases, animals dealt with food items in the order in which they were encountered on an arm. Thus, in patches with multiple distributed items, the item nearest the center was eaten or carried to the center first, the next item was eaten or carried second, and so forth. In Figure 2, the percentage of items carried is plotted as a function of order of item encountered for each patch size and each group.

The data generally agree with the prediction that raising travel time by placing barriers at the patch entrances would lower the frequency of food carrying. In all four patches, food was carried more often when no barriers were present than when barriers were present. The hypothesis that rats will carry food less readily in patches with multiple items than in patches with a single item was evaluated by comparing the percentage of initial items carried in patches of different sizes. A comparison of the percentage of items carried from the patch containing one item with the first item encountered in patches containing two, three, and four items indicates a drop in food carrying only in Group No Barriers-Distributed. In this group, the item in the one-item patch was carried significantly more often than the first item in the two-item patch $[t(5)=2.73]$ and the first item in the four-item patch $[t(5)=5.22]$ but not the first item in the three-item patch $[t(5)=1.55]$. When these three comparisons were made within the other three groups, the differences were negligible and not statistically significant.

The prediction that the last item in a patch should be carried to the center more frequently than earlier items can be examined by looking at each curve for two-, three-, and four-item patches in Figure 2. This prediction clearly is not supported by any of the curves for groups with clustered food items, since all six curves drop slightly from first item taken to last. In the distributed food items

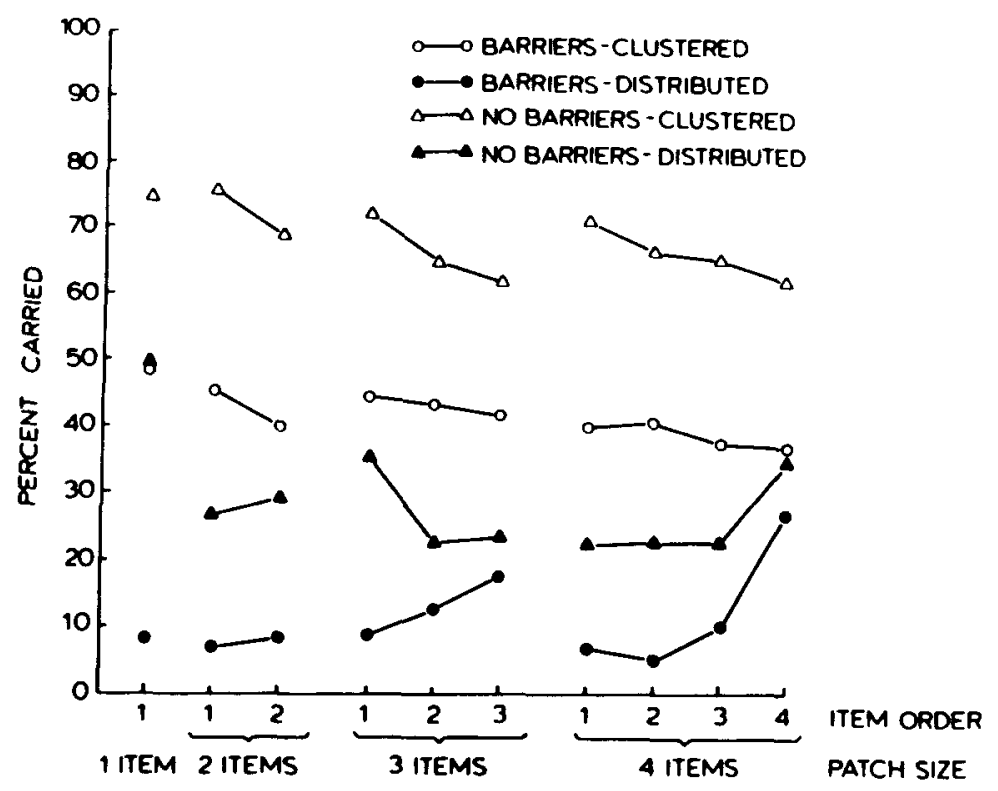

Figure 2. Percentage of food items carried in each group as a function of item order within each patch size (Experiment 1). 
groups, all of the curves rise, except that for Group No Barriers-Distributed in the three-item patch. However, the rise in food carrying over items is not significant in any of these curves, except the one for Group No BarriersDistributed in the four-item patch $[F(3,15)=3.42]$. The data provide little support overall for the notion that food carrying should increase as number of items in the patch decreases.

The data directly contradict the notion that clustering food will decrease the tendency to carry food. Food was carried more often when it was clustered than when it was distributed. Separate analyses of variance (ANOVAs) were performed on the percent carried at each patch size. For the one-item patch, a travel time $\times$ food distribution ANOVA yielded significant effects of travel time $[F(1,20)$ $=4.88]$ and food distribution $[F(1,20)=4.87]$ but not of the interaction of these variables $(F<1.0)$. In the case of two-, three-, and four-item patches, travel time $\times$ food distribution $x$ item number ANOVAs were performed on the data. The effect of travel time failed to reach significance in any of the three patches $[F(1,20) \leq 3.37]$, but the effect of food distribution was significant in all three patches $[F(1,20) \geq 5.61]$. The only significant interaction found was that of food distribution $x$ item number in the four-item patch $[F(3,60)=4.50]$. Separate ANOVAs were performed to test the effect of item number when food in the four-item patch was distributed and clustered. When food was clustered, the drop in percent carried across items encountered was not significant $[F(3,33)=1.80]$. When food was distributed, however, the percent carried increased significantly with item number $[F(3,33)=3.89]$.

Patch preferences. Rats showed no consistent pattern of visits to patches containing multiple food items. When carrying food items, some rats tended to repeatedly visit the same patch, but other rats interspersed visits to other

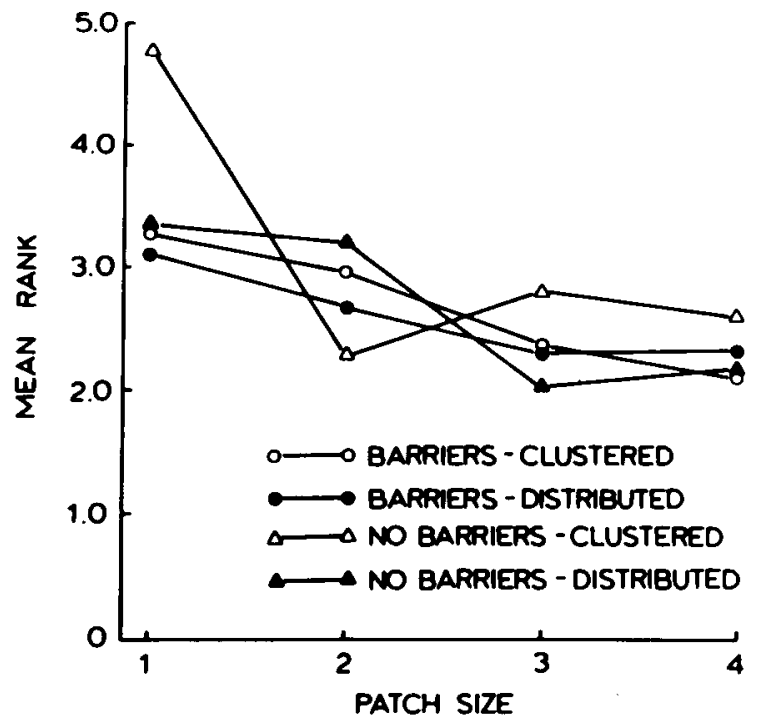

Figure 3. Mean rank of first entry into patches containing one, two, three, and four food items by rats in each group (Experiment 1).
Table 1

Percentage of Multiple-Item Loading Observations Made in Rats from Each Group in Experiment 1

\begin{tabular}{|c|c|c|c|c|c|c|}
\hline \multirow{2}{*}{$\begin{array}{l}\text { Subject } \\
\text { Number }\end{array}$} & \multicolumn{6}{|c|}{ No. Items Carried/No. Available } \\
\hline & $2 / 2$ & $2 / 3$ & $3 / 3$ & $2 / 4$ & $3 / 4$ & $4 / 4$ \\
\hline \multicolumn{7}{|c|}{ Barriers-Clustered } \\
\hline 1 & 80.0 & 80.0 & 10.0 & 80.0 & 80.0 & \\
\hline 2 & 30.0 & 40.0 & 10.0 & 20.0 & & \\
\hline \multicolumn{7}{|c|}{ No Barriers-Clustered } \\
\hline 4 & & 5.0 & & 5.0 & & \\
\hline 5 & & 5.0 & & 5.0 & & \\
\hline 6 & 10.0 & 10.0 & & 20.0 & 15.0 & \\
\hline \multicolumn{7}{|c|}{ Barriers-Distributed } \\
\hline 7 & & 10.0 & & & & \\
\hline 11 & 5.0 & 5.0 & & & & \\
\hline \multicolumn{7}{|c|}{ No Barriers-Distributed } \\
\hline 12 & & 5.0 & & & & \\
\hline 15 & 5.0 & 5.0 & & & & \\
\hline
\end{tabular}

patches between an initial and return visit to a given patch. A low level of errors or return to patches in which all food items had been taken was found. The mean number of errors per session in Groups Barriers-Clustered, BarriersDistributed, No Barriers-Clustered, and No BarriersDistributed was .62, .32, 1.48, and .76, respectively. Statistical analysis showed that rats made more errors with barriers absent than with barriers present $[F(1,20)=$ 6.79], but food distribution was not a significant factor $[F(1,20)=4.27]$.

Figure 3 displays the mean rank of first entrance into patches of different size for each group. Repeated entrances into patches were counted in the ranking, so the rank for any single patch could be greater than four. Mean rank drops as patch size increases, indicating that rats tended to visit the larger patches before the smaller patches. A travel time $\times$ food distribution $\times$ patch size ANOVA performed on mean rank yielded significant main effects of travel time $[F(1,20)=4.44]$ and patch size $[F(3,60)=18.38]$. Two interactions were significant, travel time $\times$ patch size $[F(3,60)=4.19]$ and food distribution $\times$ patch size $[F(3,60)=4.85]$. The significant effect of travel time and the significant interactions arise from the curve of Group No Barriers-Clustered, which is substantially higher than the curves for the other groups at the one-item patch size. The high rank seen at this point arises from the fact that animals in this group carried the majority of food items encountered and often entered and reentered the patches with multiple items before entering the patch with only one food item.

Multiple-item loading. Some central-place foragers are classified as multiple-prey loaders, because they carry several prey items at once (Stephens \& Krebs, 1986). It is interesting that some rats showed this behavior on the radial maze. On some occasions, a rat loaded more than one food item in its mouth and carried these items to the center of the maze. Data on multiple-item loading are shown in Table 1. The numbers at the top of each column indicate the number of items loaded over the number of 
items available, and the numbers in the table indicate the percentage of the opportunities on which multiple items were loaded. Multiple-item loading was performed by 2 rats in Group Barriers-Clustered, 3 rats in Group No Barriers-Clustered, 2 rats in Group Barriers-Distributed, and 2 rats in Group No Barriers-Distributed. Among the rats that showed multiple-item loading, the behavior was shown more frequently when food items were clustered at the end of the maze arms.

Partial carrying. Partial carrying occurred when a rat occasionally carried a food item only part of the way to the center and ate it on the alley. This behavior was seen almost exclusively in the groups with distributed food items. It appeared only twice in 1 rat in Group BarriersClustered, but it appeared in 2 animals in Group BarriersDistributed, twice in 1 rat and 17 times in the other; partial carrying was also seen in 2 rats in Group No BarriersDistributed, twice in 1 rat and eight times in the other. When rats in the distributed conditions partially carried a food item, it was almost always the final item in the patch located at the end of the arm. Of the total partial carries, $71.0 \%$ were the fourth item encountered in a fouritem patch, and $9.7 \%$ were the third item encountered in a three-item patch.

\section{Discussion}

On the basis of Lima et al.'s (1985) model of centralplace foraging, it was hypothesized that rats would tend to carry food less often in patches with multiple items than in a patch with a single item. This hypothesis was not strongly supported. Rats carried the single item found in the one-item patch more frequently than the first item encountered in multiple-item patches only in Group No Barriers-Distributed, and rats generally did not show a tendency to increase food carrying as the number of items in multiple-item patches decreased.

Rats demonstrated several interesting foraging strategies in this experiment. They tended to visit patches with larger numbers of food items earlier than those with smaller numbers of food items. However, the relationship between patch size and rank of patch entry was not as strong as that seen in some other experiments in which single loads of food varying in size were placed on different arms of a radial maze (Hulse \& O'Leary, 1982; Roberts, in press). Rats carried fewer food items when travel time was increased by barriers placed at the arm entrances, and food carrying occurred significantly more often when food was clustered at the end of an alley than when it was distributed along an alley. Finally, some rats attempted to double or triple the payoff for a single trip by carrying two or three food items to the center at one time.

Food items were placed in clusters at the end of the maze arms to test the prediction that eating in the patch would increase when multiple food items were particularly apparent. Quite contrary to this prediction, it was found that rats carried food more often when it was clustered than when it was distributed. A post hoc account of this finding can be offered in terms of the importance of the center of the maze to foraging rats. Phelps and Roberts (1989) and Roberts et al. (in press) have argued that the center of a radial maze functions as a place of safety for foraging rats because it is that single location on the maze that provides several escape routes. Centralplace foragers often carry food either to their home or to a nearby place to consume it (Barnett, 1975; Covich, 1987). Places that are near home are relatively safe, because home can be reached immediately in case a predator arrives. Combining this principle with the difference in distribution of food in the patches between the clustered and distributed conditions provides an explanation for the higher incidence of food carrying in the clustered condition. As can be seen in Figure 1, distributing food along the arms meant that most of the food items in the distributed groups were nearer the center of the maze than were the food items placed at the ends of the arms in the clustered groups. Rats may have felt safer eating items on the arms in the distributed condition because these items were closer to the center. Rats in the clustered groups may have carried items more frequently not because they were clustered, but because they were located at the farthest possible distance from the center.

Some other observations support this interpretation. The interaction between item number and food distribution in the four-item patch (seen in Figure 2) was largely caused by the increase in food carrying by rats in the distributed conditions at the fourth item. The fourth item was located at the end of the arm and should be carried to safety, whereas the other items nearer the center could often be eaten in this relatively safer territory. Another supporting piece of evidence is the pattern of partial carrying seen in several animals in the distributed groups. When these rats partially carried food items, they most frequently carried the item at the end of the four-item patch part of the distance down the arm and ate it at that point. Rats appeared to be moving food items from a position of danger to a safer position near the center.

\section{EXPERIMENT 2}

Food distribution and distance of food from the center of the maze were confounded in Experiment 1. Because clustered food always was placed on the ends of maze arms, the average distance of distributed food items was necessarily closer to the center. Although we have argued that distance of the food from the center was the critical factor leading to more food carrying in the clustered groups, it could be argued that clustering versus distribution was the critical variable, not distance. For example, it might be the case that placing food in a cluster increased the apparent size of each food item and therefore increased the tendency for rats to carry food items. We judge this account unlikely, because it cannot account for the difference between clustered and distributed conditions seen in the one-item patch in Experiment 1. Never- 
theless, we felt it important to carry out a further experiment, to examine the effect of distance from the maze center unconfounded by food distribution.

In Experiment 2, one, two, three, and four food items again were placed on the four arms of the radial maze for each of two groups of rats. In the patches with multiple food items, the food was always clustered. For one group of rats, the food clusters were placed at the ends of maze arms (Group End), and for the other group, the clusters were always placed in the middles of the maze arms (Group Middle), as is shown in the central and righthand mazes of Figure 1. If the tendency to carry food items is controlled only by food clustering, a high and equal level of food carrying should be seen in both groups. If distance of the food from the center of the maze is critical, however, significantly more food carrying should be seen in the distant group than in the near group. In addition, this experiment provided one further test of the possibility that patch size influences the tendency to carry food to the center of the maze.

\section{Method}

Subjects. Twenty-four male Long-Evans rats served as subjects. They were 100-120 days of age at the start of the experiment and were kept at approximately $85 \%$ of free-feeding weight throughout testing.

Apparatus. The radial maze and testing room from Experiment 1 were used in Experiment 2.

Procedure. The 24 rats were pretrained without barriers in the manner described in Experiment 1 and were then randomly divided into two groups, with one group designated Group Middle and the other designated Group End. Food items placed on the maze were 0.65-g cubes of cheese. Each arm of the maze was assigned one, two, three, or four food items, with the number of items assigned to an arm varying between rats but remaining constant throughout testing for each rat. The clusters of food items were placed on the ends of the arms for Group End and at distances of $40 \mathrm{~cm}$ from the center for Group Middle, as depicted in Figure 1. Each rat was allowed to forage until all of the food items were consumed for one session each day over a period of 20 days. The data were recorded in the same manner as in Experiment 1.

\section{Results}

Food carrying. In Figure 4, the percentage of food items carried is shown for Group Middle and Group End as a function of item number at each patch size. The curves clearly support the hypothesis that rats carried food more frequently from the end of an arm than from its middle. The difference between Group Middle and Group End was significant for the one-item patch $[t(22)=1.88]$, the twoitem patch $[F(1,22)=6.99]$, the three-item patch $[F(1,22)$ $=8.80]$, and the four-item patch $[F(1,22)=7.11]$.

For each of the patches containing multiple food items, there was a drop in percent carried across items consumed. Groups $x$ item number ANOVAs performed for each patch size revealed significant effects of item number for the two-item patch $[F(1,22)=6.22]$, the threeitem patch $[F(2,44)=3.38)$, and the four-item patch $[F(3,66)=3.71]$. The curves in Figure 4 suggest that these decreases in food carrying over successive items were largely parallel in Group Middle and Group End, and this impression was supported by the absence of an interaction between groups and item number $(F<1.0$ in all cases).

The data in Figure 4 also yield some support for the hypothesis that increasing patch size depresses food carrying. In the case of Group End, the percentage of initial items carried in the two-item and three-item patches did not differ significantly from the percentage carried in the

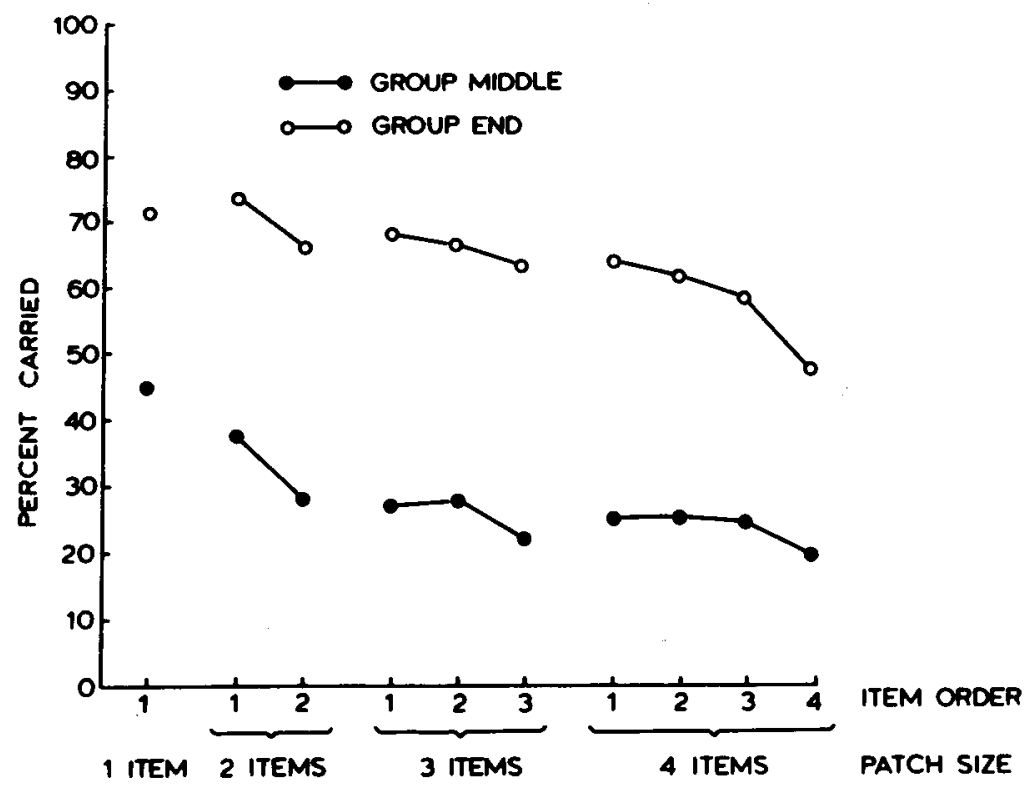

Figure 4. Percentage of food items carried in each group as a function of item order within each patch size (Experiment 2). 


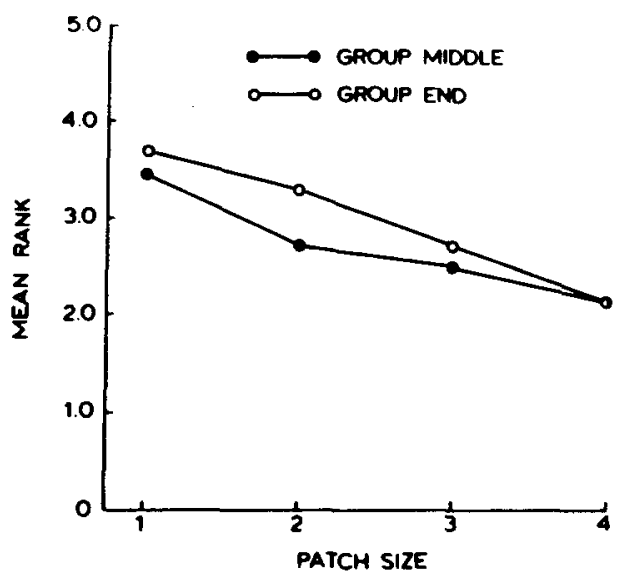

Figure 5. Mean rank of first entry into patches containing one, two, three, and four food items by rats in each group (Experiment 2).

one-item patch $(t<1.0)$, but the initial item in the fouritem patch was carried significantly less frequently than the single item in the one-item patch $[t(11)=1.89]$. In Group Middle, the drop in food carrying from the oneitem patch to the initial item of multiple-item patches was significant for both the three-item patch $[t(11)=2.81]$ and the four-item patch $[t(11)=4.44]$ but not for the twoitem patch $[t(11)=1.68]$.

Patch preferences. As in Experiment 1, rats made some errors by returning to patches they had emptied of food. The mean number of errors per session was 1.66 in Group End and 1.48 in Group Middle. The frequency of errors did not differ significantly between groups $(t<1.0)$.

Clear evidence of preference for the larger patches was found in both groups. In Figure 5, curves for both Group Middle and Group End show a monotonic decline in mean rank of patch entry as patch size increases. Statistical analysis of these curves yielded a significant effect of patch size $[F(3,66)=16.41]$, but not of group $[F(1,22)=3.84]$ or of the group $\times$ patch size interaction $(F<1.0)$.

Multiple-item loading. As in Experiment 1, multipleitem loading was seen in some rats. Table 2 shows that

Table 2

Percentage of Multiple-Item Loeding Observations Made in Rats from Each Group in Experiment 2

\begin{tabular}{|c|c|c|c|c|c|c|}
\hline \multirow{2}{*}{$\begin{array}{l}\text { Subject } \\
\text { Number }\end{array}$} & \multicolumn{6}{|c|}{ No. Items Carried/No. Available } \\
\hline & $2 / 2$ & $2 / 3$ & $3 / 3$ & $2 / 4$ & $3 / 4$ & $4 / 4$ \\
\hline \multicolumn{7}{|c|}{ Group End } \\
\hline $\begin{array}{l}22 \\
23\end{array}$ & 5.0 & 5.0 & & $\begin{array}{r}5.0 \\
10.0\end{array}$ & & \\
\hline 33 & 20.0 & 40.0 & 10.0 & 55.0 & 5.0 & 5.0 \\
\hline 36 & 75.0 & 80.0 & 5.0 & 60.0 & 15.0 & \\
\hline \multicolumn{7}{|c|}{ Group Middle } \\
\hline 24 & & 5.0 & & & & \\
\hline 25 & 10.0 & & & 20.0 & & \\
\hline 26 & & 25.0 & & & & \\
\hline 35 & 75.0 & 35.0 & 55.0 & 5.0 & 20.0 & 20.0 \\
\hline
\end{tabular}

4 rats in Group End and 4 rats in Group Middle carried more than one cube of cheese in their mouths on several occasions. Although a maximum of three food items was carried at once in Experiment 1,2 rats in Experiment 2 carried all four items from the four-item patch to the center of the maze. Rat 33 in Group End did this on one occasion, and Rat 35 in Group Middle did it on four occasions.

Partial carrying. The tendency for rats to carry items from their initial position to one on the arm nearer the center for consumption was again seen in Experiment 2. It was over twice as common an event in Group End as it was in Group Middle. Seven rats in Group End partially carried items on some days for a total of 82 instances, whereas 3 rats in Group Middle partially carried items on some days for a total of 37 instances.

\section{GENERAL DISCUSSION}

The primary purpose of these experiments was to test the prediction drawn from an optimal foraging model (Lima et al., 1985) that rats should tend to carry food to a central place of safety more often when one food item is encountered in a patch than when multiple items are encountered. The cleanest test of this prediction involves comparing the degree of food carrying seen for the first item encountered in multiple-item patches with the first (and only) item encountered in a single-item patch. The findings of Experiment 1 offered only weak support for this prediction, because the predicted difference between one-item and multiple-item patches was found in only one of four groups of subjects. The results of Experiment 2 offer stronger support. In both Group End and Group Middle, initial items were carried reliably more frequently from the one-item patch than from the larger multiple-item patches.

If it is assumed that the prediction about carrying the initial item from patches varying in size is supported by these data, a surprising paradox arises when the data for successive items encountered within patches are examined. Lima et al.'s (1985) model of optimal tradeoff between predation risk and foraging efficiency clearly predicts that the final item in a patch should be carried more often than previous items taken from the same patch. Yet the data from patches of clustered items showed consistently in both Experiments 1 and 2 that food carrying dropped monotonically from first item encountered to last. This strange contradiction between the effects of patch size on initial items and last items suggests that different processes may control food-carrying decisions made on early and late patch entries. One possible account of these results is that the level of fear needed to motivate a relatively high level of food carrying from a single-item patch is present on the first visit of the day to each patch. As patches are repeatedly visited, however, fear of the patch adapts, and the tendency to carry items to the center weakens.

In contrast with the data found with clustered food items, rats tended to increasingly carry the distributed food items encountered in Experiment 1. We suggested that this effect may have arisen from the increasing distance of distributed food items from the center of the maze and 
not from food distribution per se. It was hypothesized that rats' tendency to carry food to the center of the maze increases with distance of the food item from the center. This prediction follows from an analysis of the radial maze as a situation in which rats feel most safe in the center of the maze, and in which fear of predation increases with distance from the center. This hypothesis was tested unconfounded by food distribution in Experiment 2 by placing clusters of food at the end or the middle of maze arms. The hypothesis was fully supported by a significantly higher level of food carrying from the ends of arms than from the middle at each patch size.

In a number of ways, rats foraged in these experiments in a manner that approached optimality. In addition to the effects of patch size and distance of food from the center on food carrying already discussed, rats tended to visit patches in descending order of patch size. This finding agrees with other observations that rats learn to order visits to arms according to the amount of food contained on the arm (Hulse \& O'Leary, 1982; Roberts, in press) and with the notion that an optimal forager will maximize its rate of food gain (Stephens \& Krebs, 1986). The advantage of this strategy lies in the fact that, should a forager be forced by an intruder to fight or take flight, the amount of food gained will have been maximized up to that moment.

Finally, two other behaviors observed in these experiments suggest optimal foraging. One solution to the problem of trading off foraging efficiency against risk of predation is to carry multiple food items from the patch to a place of safety. This behavior simultaneously minimizes exposure time in the patch and achieves efficiency by eliminating the travel time necessitated by return visits. Some of the rats in these experiments used this strategy on some occasions by loading two, three, or four food items in their mouths for transportation to the center. It was a substantial feat for a rat to load four pieces of $0.65-\mathrm{g}$ cheese in its mouth and carry them to the center. In one instance, a rat loaded three pieces in its mouth and pushed the fourth piece to the center with its paws!

The other behavior of interest was the observation that some rats partially carried food to a point on the arm nearer the center of the maze and consumed it at that point.
Central-place foragers in natural settings often carry food to a location near their home, allowing rapid access to a place of safety in case a predator should appear. That rats were moving food to a perceived safer place for consumption on the radial maze is supported by the observation that partial carrying was seen far more frequently in Experiments 1 and 2 when food was encountered at the end of an arm than when it was encountered nearer the center of the maze.

\section{REFERENCES}

BARnett, S. A. (1975). The rat. Chicago: University of Chicago Press. Covich, A. P. (1987). Optimal use of space by neighboring central place foragers: When and where to store surplus resources. In L. Green \& H. Kagel (Eds.), Advances in behavioral economics (pp. 249-294). Norwood, NJ: Ablex.

Flannelly, K. J., Kemble, E. D., Horı, K. (1986). Structure, contents, and distribution of roof rat (Rattus rattus) burrows in a rain forest. Journal of Comparative Psychology, 100, 208-214.

Hulse, S. H., \& O'Leary, D. K. (1982). Serial pattern learning: Teaching an alphabet to rats. Journal of Experimental Psychology: Animal Behavior Processes, 8, 260-273.

Lima, S. L., Valone, T. J., \& Caraco, T. (1985). Foraging efficiencypredation risk trade-off in the grey squirrel. Animal Behaviour, 33 , 155-165.

Neider, L., Cagnin, M., \& Parisi, L. (1982). Burrowing and feeding behavior in the rat. Animal Behaviour, 30, 837-844.

Phelps, M. T., \&oberts, W. A. (1989). Central-place foraging by Rattus norvegicus on a radial maze. Journal of Comparative Psychology, 103, 326-338.

ROBERTS, W. A. (in press). Foraging by rats on a radial maze: Learning, memory, and decision rules. In I. Gormezano \& E. A. Wasserman (Eds.), Learning and memory: Behavioral and biological substrates. Hillsdale, NJ: Erlbaum.

Roberts, W. A., Phelps, M. T., \& Schacter, G. B. (in press). Stimulus control of central place foraging on the radial maze. In $W$. $K$. Honig \& J. G. Fetterman (Eds.), Cognitive aspects of stimulus control. Hillsdaie, NJ: Erlbaum.

SChacter, G. B., Phelps, M. T., Brodbeck, D. R., Mogenson, G. J., \& Roberts, W. A. (1991). Disruption of central-place foraging in the rat following lesions of the dorsomedial thalamic nucleus. Psychobiology, 19, 91-99.

STEPHENS, D. W., \& KREBS, J. R. (1986). Foraging theory. Princeton, NJ: Princeton University Press.

(Manuscript received January 21, 1991; revision accepted for publication January 22, 1992.) 